\title{
The Majorana Demonstrator: A Search for Neutrinoless Double-beta Decay of Germanium-76
}

\author{
A.G. Schubert ${ }^{\mathrm{a}}$, E. Aguayo ${ }^{\mathrm{b}}$, F.T. Avignone III ${ }^{\mathrm{c}, \mathrm{d}}$, H.O. Back ${ }^{\mathrm{e}, \mathrm{f}}$, A.S. Barabash ${ }^{\mathrm{g}}$,
} M. Bergevin ${ }^{\mathrm{h}}$, F.E. Bertrand ${ }^{\mathrm{d}}$, M. Boswell ${ }^{\mathrm{i}}$, V. Brudanin ${ }^{\mathrm{j}}$, M. Busch ${ }^{\mathrm{k}, \mathrm{f}}$, Y-D. Chan ${ }^{\mathrm{h}}$, C.D. Christofferson ${ }^{1}$, J.I. Collar ${ }^{\mathrm{m}}$, D.C. Combs ${ }^{\mathrm{e}, \mathrm{f}}$, R.J. Cooper ${ }^{\mathrm{d}}$, J.A. Detwiler ${ }^{\mathrm{h}}$, J. Leon ${ }^{\mathrm{a}}$, P.J. Doe ${ }^{\mathrm{a}}$, Yu. Efremenko ${ }^{n}$, V. Egorov ${ }^{\mathrm{j}}$, H. Ejiri ${ }^{\circ}$, S.R. Elliott ${ }^{\mathrm{i}}$, J. Esterline ${ }^{\mathrm{k}, \mathrm{f}}$, J.E. Fast ${ }^{\mathrm{b}}$, N. Fields ${ }^{\mathrm{m}}$, P. Finnerty ${ }^{\mathrm{p}, \mathrm{f}}$, F.M. Fraenkle ${ }^{\mathrm{p}, \mathrm{f}}$, V.M. Gehman ${ }^{\mathrm{i}}$, G.K. Giovanetti ${ }^{\mathrm{p}, \mathrm{f}}$, M.P. Green ${ }^{\mathrm{p}, \mathrm{f}}$, V.E. Guiseppe ${ }^{\mathrm{q}}$, K. Gusey $^{\mathrm{j}}$, A.L. Hallin ${ }^{\mathrm{r}}$, R. Hazama ${ }^{\mathrm{a}}$, R. Henning ${ }^{\text {p,f }}$, A. Hime ${ }^{\mathrm{i}}$, E.W. Hoppe ${ }^{\mathrm{b}}$, M. Horton ${ }^{1}$, S. Howard $^{1}$, M.A. Howe ${ }^{\mathrm{p}, \mathrm{f}}$, R.A. Johnson ${ }^{\mathrm{a}}$, K.J. Keeter ${ }^{\mathrm{s}}$, M.E. Keillor ${ }^{\mathrm{b}}$, C. Keller ${ }^{\mathrm{q}}$, J.D. Kephart ${ }^{\mathrm{b}}$, M.F. Kidd ${ }^{\mathrm{i}}$, A. Knecht ${ }^{\mathrm{a}}$, O. Kochetov ${ }^{\mathrm{j}}$, S.I. Konovalov ${ }^{\mathrm{g}}$, R.T. Kouzes ${ }^{\mathrm{b}}$, B. LaFerriere ${ }^{\mathrm{b}}$, B.H. LaRoque ${ }^{\mathrm{i}}$, L.E. Levinere,f , J.C. Loach ${ }^{\mathrm{h}}$, S. MacMullin ${ }^{\mathrm{p}, \mathrm{f}}$, M.G. Marino ${ }^{\mathrm{a}}$, R.D. Martin ${ }^{\mathrm{h}}$, D.-M. Mei ${ }^{\mathrm{q}}$, J. Merriman ${ }^{\mathrm{b}}$, M.L. Miller ${ }^{\mathrm{a}}$, L. Mizouni ${ }^{\mathrm{c}, \mathrm{b}}$, M. Nomachi ${ }^{\mathrm{o}}$, J.L. Orrell ${ }^{\mathrm{b}}$, N. Overman ${ }^{\mathrm{b}}$, D.G. Phillips II ${ }^{\mathrm{p}, \mathrm{f}}$, A.W.P. Poon ${ }^{\mathrm{h}}$, G. Perumpilly ${ }^{\mathrm{q}}$, G. Prior ${ }^{\mathrm{h}}$, D.C. Radford ${ }^{\mathrm{d}}$, K. Rielage ${ }^{\mathrm{i}}$, R.G.H. Robertson ${ }^{a}$, M.C. Ronquest ${ }^{i}$, T. Shima ${ }^{o}$, M. Shirchenko ${ }^{j}$, K.J. Snavely ${ }^{p, f}$, V. Sobolev ${ }^{\mathrm{l}}$, D. Steele ${ }^{\mathrm{i}}$, J. Strain ${ }^{\mathrm{p}, \mathrm{f}}$, K. Thomas ${ }^{\mathrm{q}}$, V. Timkin ${ }^{\mathrm{j}}$, W. Tornow ${ }^{\mathrm{k}, \mathrm{f}}$, I. Vanyushin ${ }^{\mathrm{g}}$, R.L. Varner ${ }^{\mathrm{d}}$, K. Vetter ${ }^{\mathrm{t}, \mathrm{h}}$, K. Vorren ${ }^{\mathrm{p}, \mathrm{f}}$, J.F. Wilkerson ${ }^{\mathrm{p}, \mathrm{d}}$, B.A. Wolfe ${ }^{\mathrm{a}}$, E. Yakushev ${ }^{\mathrm{j}}$, A.R. Young ${ }^{\mathrm{e}, \mathrm{f}}$, C.-H. Yu ${ }^{\mathrm{d}}$, V. Yumatov ${ }^{\mathrm{g}}$ and C. Zhang ${ }^{\mathrm{q}}$

${ }^{a}$ Center for Experimental Nuclear Physics and Astrophysics and Department of Physics, University of Washington, Seattle, WA, USA

${ }^{b}$ Pacific Northwest National Laboratory, Richland, WA, USA

${ }^{c}$ Department of Physics and Astronomy, University of South Carolina, Columbia, SC, USA ${ }^{d}$ Oak Ridge National Laboratory, Oak Ridge, TN, USA

${ }^{e}$ Department of Physics, North Carolina State University, Raleigh, NC, USA

${ }^{f}$ Triangle Universities Nuclear Laboratory, Durham, NC, USA

${ }^{g}$ Institute for Theoretical and Experimental Physics, Moscow, Russia

${ }^{h}$ Nuclear Science Division, Lawrence Berkeley National Laboratory, Berkeley, CA, USA ${ }^{i}$ Los Alamos National Laboratory, Los Alamos, NM, USA

${ }^{j}$ Joint Institute for Nuclear Research, Dubna, Russia

${ }^{k}$ Department of Physics, Duke University, Durham, NC, USA

${ }^{l}$ South Dakota School of Mines and Technology, Rapid City, SD, USA

${ }^{m}$ Department of Physics, University of Chicago, Chicago, IL, USA

${ }^{n}$ Department of Physics and Astronomy, University of Tennessee, Knoxville, TN, USA

${ }^{\circ}$ Research Center for Nuclear Physics and Department of Physics, Osaka University, Ibaraki, Osaka, Japan

${ }^{p}$ Department of Physics and Astronomy, University of North Carolina, Chapel Hill, NC, USA

${ }^{q}$ Department of Earth Science and Physics, University of South Dakota, Vermillion, SD, USA

${ }^{r}$ Centre for Particle Physics, University of Alberta, Edmonton, AB, Canada

${ }^{s}$ Department of Physics, Black Hills State University, Spearfish, SD, USA

${ }^{t}$ Alternate address: Department of Nuclear Engineering, University of California, Berkeley, CA, USA 


\begin{abstract}
.
The observation of neutrinoless double-beta decay would determine whether the neutrino is a Majorana particle and provide information on the absolute scale of neutrino mass. The MAJORANA Collaboration is constructing the DEMONSTRATOR, an array of germanium detectors, to search for neutrinoless double-beta decay of ${ }^{76} \mathrm{Ge}$. The DEMONSTRATOR will contain $40 \mathrm{~kg}$ of germanium; up to $30 \mathrm{~kg}$ will be enriched to $86 \%$ in ${ }^{76} \mathrm{Ge}$. The DEMONSTRATOR will be deployed deep underground in an ultra-low-background shielded environment. Operation of the DEMONSTRATOR aims to determine whether a future tonne-scale germanium experiment can achieve a background goal of one count per tonne-year in a 4-keV region of interest around the ${ }^{76} \mathrm{Ge}$ neutrinoless double-beta decay Q-value of $2039 \mathrm{keV}$.
\end{abstract}

Keywords: germanium, low-background, neutrinoless double-beta decay PACS: $29.40 . \mathrm{Wk}, 23.40 . \mathrm{Bw}, 14.60 . \mathrm{Pq}, 29.30 . \mathrm{Kv}$

\title{
INTRODUCTION
}

Observation of neutrinoless double-beta decay $(0 v \beta \beta)$ would determine the Majorana nature of the neutrino and would demonstrate that lepton number is not conserved. Additionally, the measurement of a $0 v \beta \beta$ rate will provide information about the absolute scale of neutrino mass. The MAJORANA Collaboration [1] will search for neutrinoless double beta decay with an array of germanium detectors enriched in the $0 v \beta \beta$ candidate isotope ${ }^{76} \mathrm{Ge}$.

The MAJORANA and GERDA [2] Collaborations are independently investigating technologies for deployment of ultra-low-background germanium detector arrays as research and development toward a future collaborative tonnescale $0 v \beta \beta$ experiment. As part of this effort, MAJORANA is constructing the DEMONSTRATOR, a germanium detector array, with the goal of testing a recent claimed observation of $0 v \beta \beta$ [3] and determining the background rates achievable with the proposed compact shield design. For a tonne-scale experiment, MAJORANA and GERDA have a background goal of one count per tonne-year after analysis cuts in a narrow energy region surrounding the double-beta decay endpoint at $2039 \mathrm{keV}$. The energy region of interest (ROI) is expected to be approximately $4 \mathrm{keV}$ wide, with the actual width depending on detector resolution. This background goal is approximately 100 times more stringent than background rates observed in previous low-background germanium experiments [4], [5].

\section{THE MAJORANA DEMONSTRATOR}

The Demonstrator will contain $40 \mathrm{~kg}$ of germanium semiconductor diode detectors, of which at least $20 \mathrm{~kg}$ and up to $30 \mathrm{~kg}$ will be enriched to $86 \%$ in ${ }^{76} \mathrm{Ge}$. The DEMONSTRATOR will be located at the 4850 -foot level of Sanford Underground Research Facility in Lead, SD. An array of enriched detectors is expected to begin operation in 2013. Germanium detectors will be housed in two vacuum cryostats constructed from electroformed copper and cooled with liquid nitrogen. The detectors will be mounted in strings, rigid columns of detectors suspended from an electroformed copper cold plate. Each cryostat will contain seven strings of five detectors in a close-packed geometry.

The DemOnSTRATOR cryostats will be surrounded by several layers of shielding, shown in Fig. 1. In order of proximity to the detectors, these layers will consist of electroformed copper, commercial copper, lead, a radon exclusion volume, an active muon scintillator veto, and polyethylene neutron moderator. A background count rate at or below four counts per tonne-year in the DEMONSTRATOR ROI would scale to the background goal of one count per tonne-year in the ROI in a tonne-scale ${ }^{76} \mathrm{Ge}$ experiment.

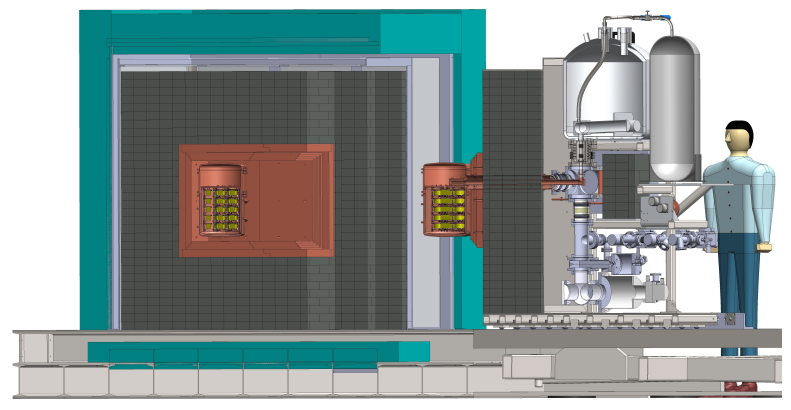

FIGURE 1. A drawing of the Majorana Demonstrator, showing insertion of a cryostat module into the shield. 


\section{$0 v \beta \beta$ DETECTION AND BACKGROUND IDENTIFICATION TECHNIQUES}

A $0 v \beta \beta$ signal would appear as a peak at the Q-value in the energy spectrum collected by the DEMONSTRATOR. The sensitivity of the DEMONSTRATOR is limited by background contributions in the ROI; any radiation with energy greater than the Q-value could potentially create a background in the ROI. Backgrounds are expected from in-situ cosmogenic flux, long-lived unstable cosmogenically activated contaminants such as ${ }^{68} \mathrm{Ge}$ in the detectors and ${ }^{60} \mathrm{Co}$ in the copper, and primordial radioimpurities, such as ${ }^{40} \mathrm{~K},{ }^{238} \mathrm{U}$, and ${ }^{232} \mathrm{Th}$ decay products. Only materials certified by ongoing assay and simulation campaigns will be used. Component mass is minimized to reduce contaminants. Close packing of the detectors enhances effectiveness of a crystal-to-crystal granularity tag. Measurements in dedicated thermal and electronics test cryostats have refined the designs of front-end electronics and detector mounts.

MAJORANA has chosen p-type point contact (PPC) germanium detectors for their superior background rejection capabilities. The low energy threshold achievable with these detectors should allow identification of certain backgrounds that are time-correlated with low-energy events and will provide sensitivity to some dark matter candidates. Enriched germanium detectors provide an advantageous source-as-detector configuration with extremely high efficiency for detecting potential $0 v \beta \beta$ decays. Germanium detectors exhibit excellent energy resolution, approximately $0.2 \%$ FWHM at the $0 v \beta \beta$ Q-value, which allows the definition of a narrow ROI and minimizes contributions of backgrounds.

Pulse-shape analysis can distinguish an energy deposit occurring in a single site in a detector, characteristic of the $0 v \beta \beta$ signal, from multi-site interactions characteristic of gamma backgrounds. This distinction can be made with with high efficiency for accepting single-site events and rejecting backgrounds [6]. A gamma ray from a background source may deposit energy in one detector, exit the detector, and deposit energy in another detector. These background events can be identified with the granularity tag. Some radioactive decays, including the decay of ${ }^{68} \mathrm{Ge}$ to ${ }^{68} \mathrm{Ga}$, may be identified not only by their multiple-site nature, but also by analysis of correlations in time, energy, and location.

Ultra-clean fabrication efforts are producing components for the DEMONSTRATOR. Copper electroforming is underway at underground laboratories at a shallow site at Pacific Northwest National Laboratory in Richland, WA, and at the 4850-foot level of Sanford Underground Research Facility. A prototype Parylene deposition system has produced low-background Parylene-coated copper ribbon cable for readout of detector electronics.

Operating for five years, the DEMONSTRATOR will achieve only a fraction of a tonne-year of exposure, and less than one count is expected in the ROI. A thorough understanding of the full DEMONSTRATOR background energy spectrum will be required to predict the background count rate in the ROI of a tonne-scale ${ }^{76} \mathrm{Ge}$ experiment. By measuring the energy spectrum in a window up to $250 \mathrm{keV}$ wide surrounding the $0 v \beta \beta \mathrm{Q}$-value, a sensitive measurement can be made with Demonstrator and extrapolated to the tonne scale. The Monte-Carlo physics simulation package MAGE [7] is used to simulate DEMONSTRATOR detector response to backgrounds. MAGE results are combined with information from the material assay campaign to predict the DEMONSTRATOR background energy spectrum.

\section{CONCLUSIONS}

MAJORAna is constructing the DemOnSTRATOR, a 40-kg germanium detector array to search for $0 v \beta \beta$ of ${ }^{76} \mathrm{Ge}$. Efforts in development of ultra-low-background electronics and cabling, fabrication of radiopure components, material assay, and modeling of backgrounds are underway. The DEMONSTRATOR should confirm or refute a previous claim of $0 v \beta \beta$ observation and will determine whether a background goal of one count per tonne-year is achievable for a tonne-scale experiment. The DEMONSTRATOR is expected to operate with enriched detectors in 2013.

\section{REFERENCES}

1. C. Aalseth, et al., Nuclear Physics B - Proceedings Supplements 217, 44 - 46 (2011).

2. S. Schönert, et al., Nuclear Physics B - Proceedings Supplements 145, 242 - 245 (2005).

3. H. Klapdor-Kleingrothaus, and I. Krivosheina, Mod.Phys.Lett. A21, 1547-1566 (2006).

4. H. Klapdor-Kleingrothaus, et al., The European Physical Journal A - Hadrons and Nuclei 12, 147-154 (2001).

5. C. E. Aalseth, et al., Phys. Rev. D 65, 092007 (2002).

6. R. Cooper, et al., Nucl. Instrum. Meth. A 629, 303 - 310 (2011).

7. M. Boswell, et al., Nuclear Science, IEEE Transactions on 58, 1212 -1220 (2011). 